\title{
Sliding weight supports for W7-X magnet system: structural aspects
}

\author{
V. Bykov ${ }^{1}$, J. Fellinger ${ }^{1}$, K. Egorov' ${ }^{2}$, F. Schauer ${ }^{1}$, M. Köppen ${ }^{1}$ and H. Jenzsch ${ }^{1}$ \\ ${ }^{1}$ Max-Planck-Institut f"ur Plasmaphysik, Teilinstitut Greifswald, Wendelsteinstraße 1, 17491 \\ Greifswald, Germany \\ ${ }^{2}$ ITER Organization, Route de Vinon sur Verdon, 13115 St Paul lez Durance, France \\ E-mail: victor.bykov@ipp.mpg.de
}

\section{Abstract}

The Wendelstein 7-X (W7-X) stellarator is presently under commissioning at the Max-Planck-Institut für Plasmaphysik in Greifswald. The coil system consisting of 70 superconducting coils of seven different types is supported by a massive central support structure (CSS), and thermally protected by the cryostat. The magnet system weight is borne by supports which are bolted to the cold CSS. These ten so-called cryo-legs penetrate through the cryostat wall to the warm machine base. The design of the cryo-legs incorporates glass-reinforced plastic tubes to guarantee relatively small thermal conductivity. In order to ensure free thermal shrinkage of the magnet system and to reduce stresses in the cryo-legs, sliding and rotating bearings are used as interfaces to the machine base. Tie-rods between the machine base and the warm ends of the cryo-legs prevent toroidal rotation of the magnet system, as well as any other horizontal shifts due to asymmetric loads. The assembly of the magnet system introduced some vertical imperfections in the cryo-leg positions causing considerable additional internal stresses which were not considered during the design stage. In addition, originally not planned trim coils induce unsymmetrical cyclic loads. Therefore, the previously used method to analyse one magnet system module with periodical boundary conditions is not applicable. Consequently, a model of the complete magnet system, including all five modules, was created and analysed. Fatigue analyses of the cryo-legs under the new cyclic loads, applied on top of the approximately 100 t static weight, have been performed in order to evaluate the lifetime. The paper presents the progress in structural analyses of the W7-X magnet system under the as-built conditions, loads due to the trim coil operation, and results of the weight support fatigue analysis.

Keywords: magnet system, support, sliding, stellarator, Wendelstein 7-X, finite element analysis, fusion

\section{Abbreviations}

CSS central support structure

DEMO DEMOnstration reactor

EM electromagnetic

FE finite element

FFHR Stellarator Reactor Project, Japan

GRP glass-reinforced plastic

HI high iota

HM half module

ITER Tokamak Project ITER in Cadarache, 


\section{France}

LHD Stellarator in Toki, Japan

$M B$ machine base

MO MOdule

MS magnet system

RT room temperature

TC trim coil

WP winding pack

\section{Introduction}

Conception of a superconducting magnet system (MS) support structure for large fusion machines is always a challenge. The problem is even more complicated for stellarators with the larger main radius in comparison with tokamaks of the same plasma volume. The support system has to

- provide free contraction of the MS during cool-down;

- withstand significant EM loads without structural problems;

- keep conductive heat loads on the cryogenic system low;

- be stable enough to minimize deflections of MS components; and optionally

- to be adjustable to compensate position errors.

Two main design concepts are followed up in present fusion devices:

1. Flexible supports with MS shrinkage compensation by elastic deformation (e.g. LHD, ITER, IPP stellarator reactor concept HELIAS-5B, FFHR [1], etc),

2. Sliding/hinge supports with different type of bearings (W7-X, JT60-SA).

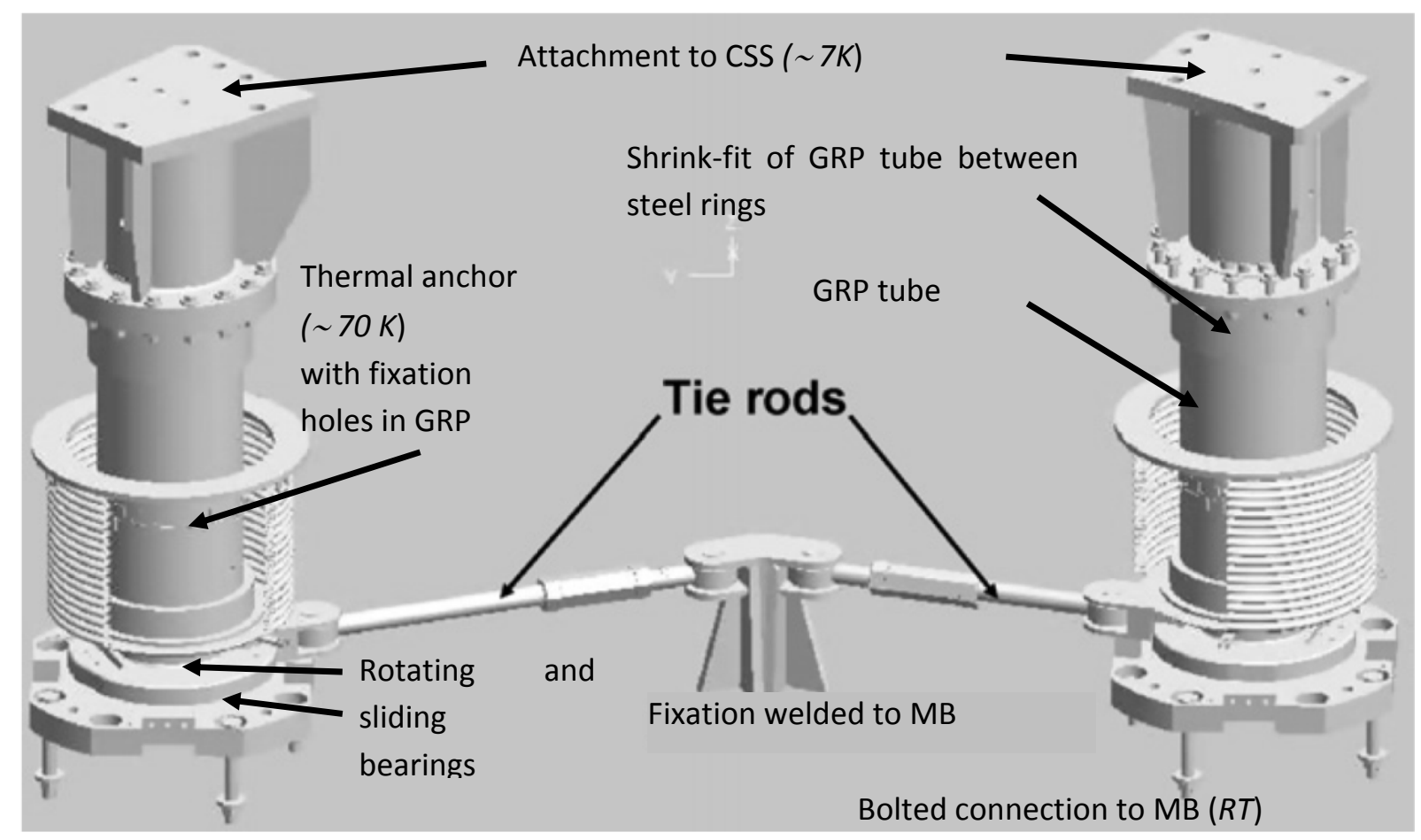

FIG. 1. Schematic view of cryo-legs of two neighboring half-modules from torus centre [2]. 
The second option is also in use for vacuum vessels of many fusion devices (e.g. ITER) to accommodate thermal expansion due to baking of vessels well above $100 \cdot \mathrm{C}$.

In addition, the supports can be divided in two main classes by the approach for heat conductance:

1. long stainless steel supports with thermal anchors (e.g. LHD, ITER, HELIAS-5B, etc),

2. glass-reinforced plastic (GRP) based support with optional thermal anchors (FFHR, W7-X).

The W7-X MS supports are bolted to the massive central support structure (CSS) at approximately $3.5 \mathrm{~m}$ radial distance from the machine axis (see figures 1 and 2). The cryo-legs reach down through the wall of the vacuum tight cryostat to the warm machine base (MB). Small thermal conductivity to keep thermal losses at an acceptable level is guaranteed by GRP tubes incorporated in the cryo-leg structure, and the thermal anchor at $50-70 \mathrm{~K}$ in the middle.

Free thermal shrinkage of the MS of radially $\approx 12 \mathrm{~mm}$ from room temperature $(\mathrm{RT})$ down to $\sim 4 \mathrm{~K}$, and further additional sliding by $\approx 10 \mathrm{~mm}$ due to amplification of CSS deformation in the cryo-leg attachment area under EM load, is provided by sliding and rotating bearings at the cryo-leg interfaces to the MB. The bearings are accessible to monitor sliding behaviour and to react in case of problems. Moreover, the chosen system of bearings with low (less than $5 \%$ ) friction keeps stress levels in the sensitive GRP tube low. The global structural analyses using one 72. module of the fivefold symmetric MS with cyclic boundary conditions under nine reference EM load cases resulted in the two extreme design load cases for the cryo-legs: $1 \mathrm{MN} / 156 \mathrm{kN}$ and $10 \mathrm{kN} / 50 \mathrm{kN}$ applied at the bottom part at sliding interface in vertical/horizontal directions respectively [3]. The design was confirmed by local static analyses of shrink-fit, cool-down, and of the mentioned design load cases, as well as by cyclic mechanical tests of the GRP at $77 \mathrm{~K}$ with corresponding FE simulations.

Assembly of the W7-X MS showed that slight changes of the cryo-leg vertical position on the order of $1 \mathrm{~mm}$ resulted in considerable additional loads and internal stresses which were not considered during the design stage. This observation triggered a reconsideration of the adjustment procedure and its limitations.

In addition, further in-depth analyses of the MS operation and field errors indicated the necessity to introduce five trim coils (TC) of two different types (type A and B) [4]. They are attached to the outer cryostat vessel and will introduce unbalanced forces and moments on the main MS and its cryolegs.

The trim coils are designed for 60000 pulses during the W7-X operational life at different levels of the MS main field which can reach $\sim 3 \mathrm{~T}$ on the plasma axis; however most of the physics programme will be carried out at $\sim 2.5 \mathrm{~T}$.

\section{FE analysis of additional loads on the cryo-legs and tie-rods}

Neither module position deviations nor trim coil operation are module-wise symmetric, so these cases have to be studied on 360. global models of the MS (see figures 2 and 3). Due to the non-linear behaviour of the MS caused by the presence of contact support elements, flanges with possibilities to open, sliding surfaces and gaps, the decomposition and superposition of impacts and responses using one module are not possible. In addition, several numerical study iterations had to be made to find a reasonable compromise between computational time and accuracy. As a result, convergence 
has been improved to provide the required precision in the tie-rod forces estimation. The two independent 360. FE models in ANSYS and ABAQUS [5] were used due to the criticality of the cryo-leg issues [6] to benchmark results and conclusions. The ABAQUS 360 model (see figure 2(b)), originally dedicated to the analysis of assembly steps [7], has been recently extended into a full operational model. Due to highly time consuming calculations it was decided to fix, by bonding, some contacts inside the models which were considered as not important for the tasks. Moreover, in the ABAQUS model more contacts were suppressed in comparison with the ANSYS one which was finally found fully acceptable for the task.

To cover the possible range of the bearing friction factor variation it was decided to have zero friction in the ABAQUS model and to keep the design friction factor of 0.1 in the ANSYS one. Change of the force application directions to represent possible opposite currents in the trim coils is another feature of the analysis verification.

The above mentioned features of two independent FE models result in some acceptable deviation of absolute displacement values, presented in figure 4 . 
(a)

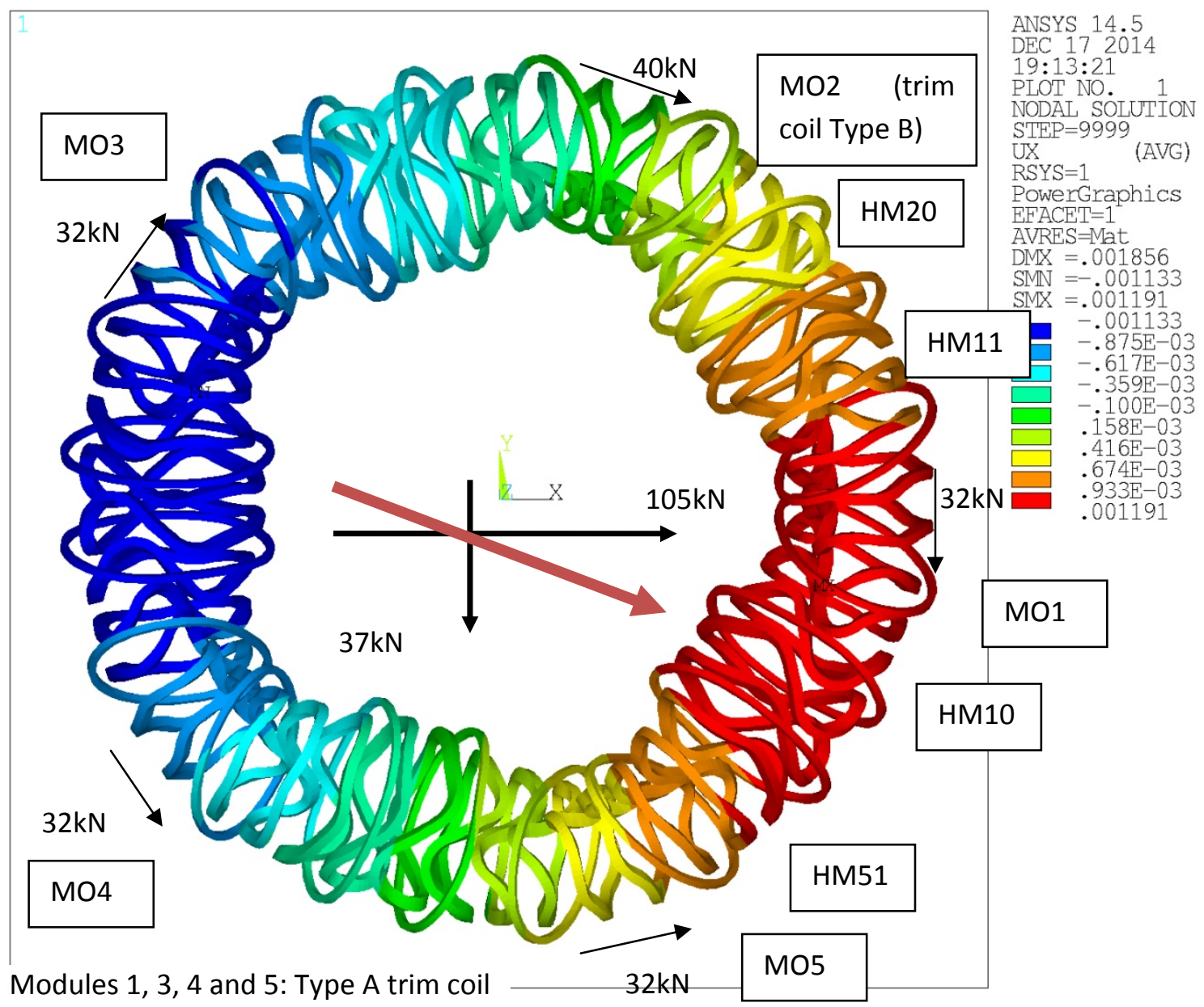

(b)

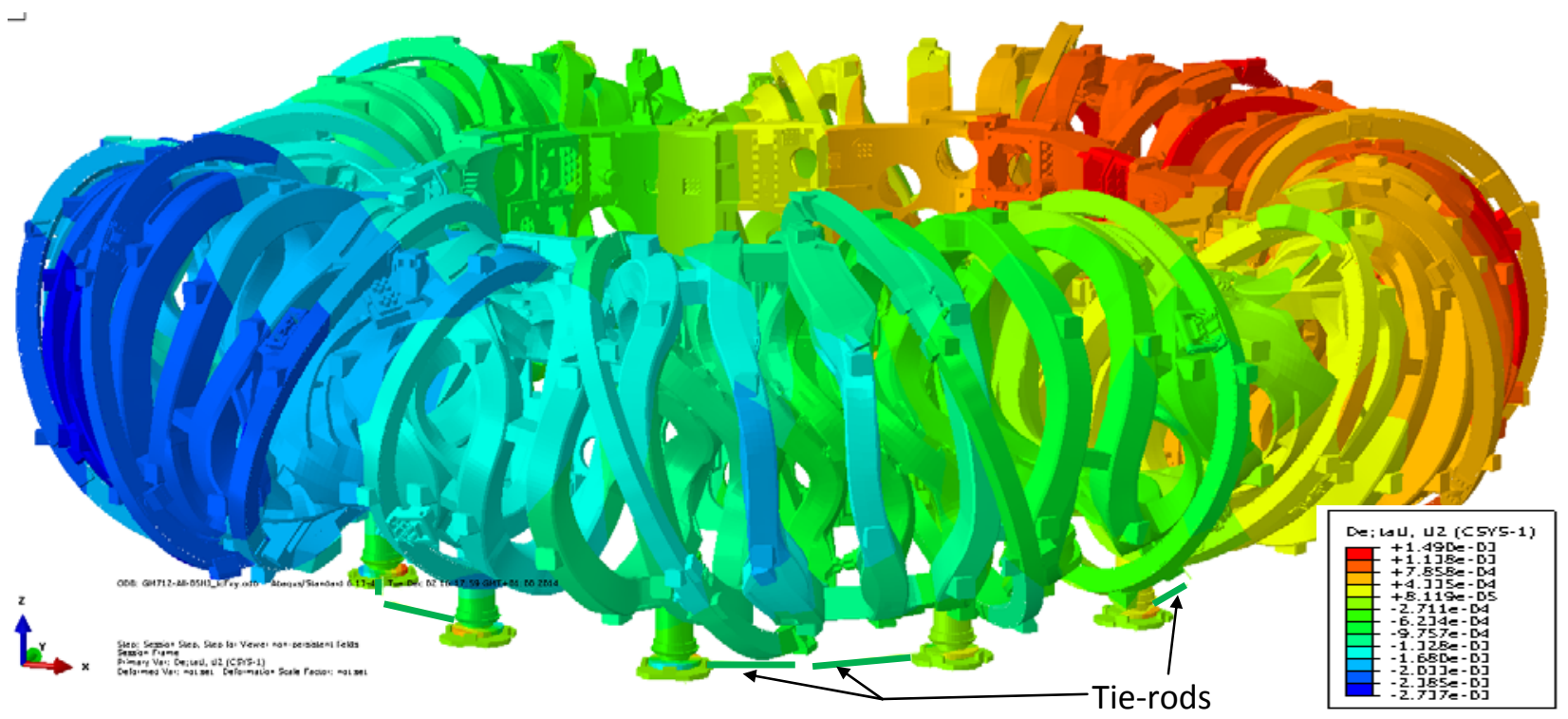

FIG. 2. (a) Asymmetry of winding pack displacements in radial direction introduced by trim coil operation (case Fxy, ANSYS); in m. Forces per module are shown schematically outside corresponding modules and total forces on magnet system shown in the middle. (b) Asymmetry of magnet system 
displacements in toroidal direction, introduced by trim coil operation (case Fxy, ABAQUS); in m. Tierods are emphasized by additional lines.

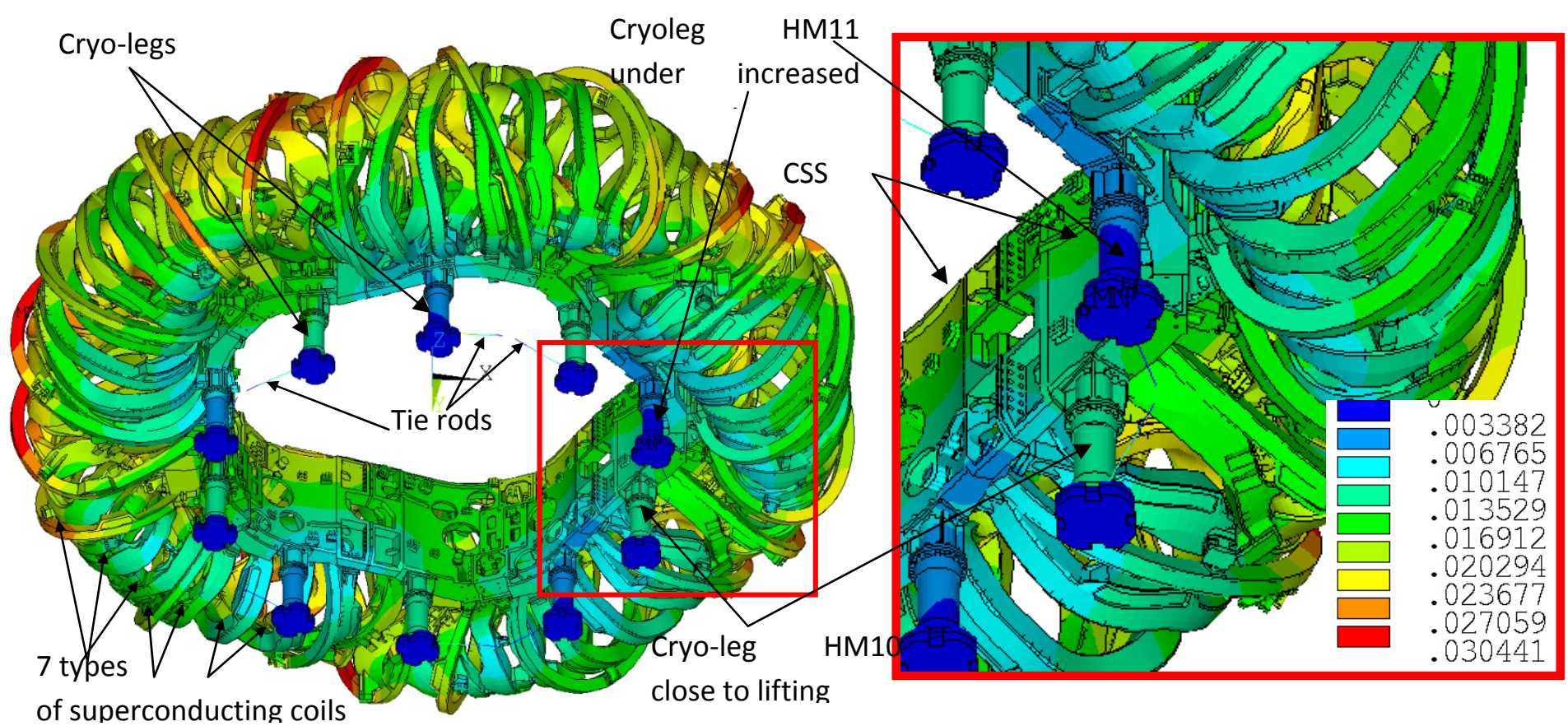

FIG. 3. Displacements of magnet system $(\mathrm{m})$ under EM loads during $\mathrm{HI}$ plasma regime and trim coil operation (case Fxy, ANSYS)

\subsection{Trim coil operations}

Trim coil load case selection has been performed for maximal forces in the tie-rods which correspond to the horizontal loads on the cryo-leg bottom ends. One plasma regime-high iota ( $\mathrm{HI})$ - has been chosen with all trim coils activated to their maximum level in different patterns to aggregate maximum horizontal force (case Fxy), torsion moment around the torus axis (case Mz), and rocking moment (case Mxy) on the MS (see table 1 and figure 2 for case Fxy). This selection is called 'extreme' in further discussions.

Displacement results are important confirmations of the MS stability under operational loads including trim coils. Figure 2 indicates that the chosen operation regime introduces significant(> \pm 2 $\mathrm{mm}$ ) asymmetry in the MS which could not be neglected in the error field assessment. A typical example for the differences in displacements (up to $3.2 \mathrm{~mm}$ ) of nonplanar coils located in the middle of the modules (type 1 coils) is shown in figure 4. The impact of this asymmetry on the error field is assessed [8] with the conclusion that the capacity of the trim coils is sufficient to mitigate additional deviations introduced by themselves. Due to CSS deformation under EM forces the dead weight of the $\mathrm{MS}$ is shifted from a rather even distribution over the cryo-legs from the half-modules $\mathrm{HMxO}$ to double values on supports attached to the odd-numbered HMx1 (see table 2). EM forces caused by the trim coil extreme case result in marginal increasing of the compressive force $(<10 \%)$ in the loaded cryo-legs, but provoke further lifting of the other cryo-legs ( $\mathrm{HMxO}$, see table 2 and figure 3 ). The resulting forces in the tie-rods are also summarized in table 2. Due to the non-linear behaviour of the FE model and the fact that tie-rod forces are almost negligible in comparison with overall forces in the MS there are small asymmetries visible during cool-down. 

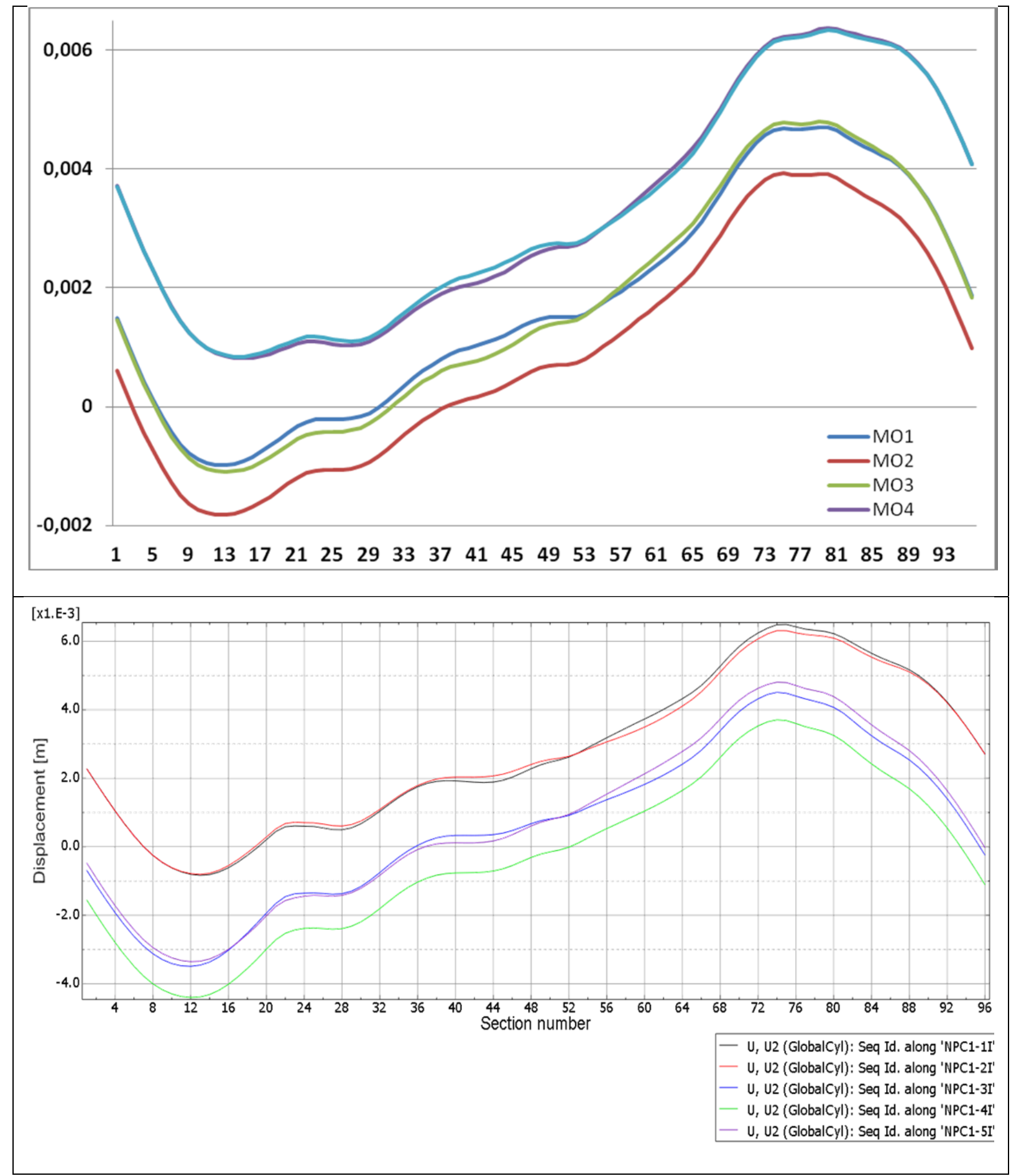

FIG. 4. Toroidal displacements along Type 1 coils of different modules $(m)$ under EM loads during HI plasma regime and trim coil operation (case Fxy, as specified in Table I). ANSYS - top, ABAQUS bottom. 


\subsection{Vertical raising of cryo-leg}

During the assembly of the MS some vertical imperfections in the cryo-leg positions were noted during monitoring process. The introduced deviations cause considerable additional internal stresses which were confirmed by dedicated analysis, and they were therefore corrected. In addition, it was realized that the vertical change of module position is covered by the design of the cryo-legs, but the corresponding load changes were not considered in detail so far. Now relatively complex analyses have been carried out to account for the observed $1 \mathrm{~mm}$ inaccuracy, and to indicate potential problems with module adjustment. Results presented in [6] have been confirmed with the ABAQUS FE model. The results of test calculations with $1 \mathrm{~mm}$ raising of the arbitrary chosen warm support, the cryo-leg HM11, shows $\sim 20 \%$ increasing of the compressive force, and provokes unloading of the adjacent cryo-legs $\mathrm{HM} 10$ and $\mathrm{HM} 20$ as shown in figure 5. The estimated increasing in the forces is covered by new design loads (see section 2.3 ).

TABLE I Trim coil current distribution and unbalanced forces/moments on magnet system

\begin{tabular}{|c|c|c|c|c|c|c|c|c|}
\hline & \multicolumn{5}{|c|}{ Maximum current in corresponding trim coils } & & & \\
\hline \multirow[t]{2}{*}{$\begin{array}{l}\text { Load } \\
\text { cases }\end{array}$} & $\begin{array}{l}\text { MO1 } \\
\text { Type A }\end{array}$ & $\begin{array}{l}\text { MO2 } \\
\text { Type B }\end{array}$ & $\begin{array}{l}\text { MO3 } \\
\text { Type A }\end{array}$ & $\begin{array}{l}\text { MO4 } \\
\text { Type A }\end{array}$ & $\begin{array}{l}\text { MO5 } \\
\text { Type A }\end{array}$ & \multicolumn{3}{|c|}{$\begin{array}{l}\text { Critical component } \\
\text { values as estimated }\end{array}$} \\
\hline & $\begin{array}{l}\text { ANSYS } \\
\text { ABAQUS }\end{array}$ & $\begin{array}{l}\text { ANSYS } \\
\text { ABAQUS }\end{array}$ & $\begin{array}{l}\text { ANSYS } \\
\text { ABAQUS }\end{array}$ & $\begin{array}{l}\text { ANSYS } \\
\text { ABAQUS }\end{array}$ & $\begin{array}{l}\text { ANSYS } \\
\text { ABAQUS }\end{array}$ & $\begin{array}{l}\text { Fxy, } \\
\text { kN }\end{array}$ & $\begin{array}{l}\mathrm{Mz} \\
\mathrm{kN} \cdot \mathrm{m}\end{array}$ & $\begin{array}{l}\mathrm{Mxy} \\
\mathrm{kN} \cdot \mathrm{m}\end{array}$ \\
\hline $\mathrm{Fxy}^{1}$ & $\begin{array}{c}1 \\
-1\end{array}$ & $\begin{array}{l}1 \\
-1\end{array}$ & $\begin{array}{l}1 \\
1\end{array}$ & $\begin{array}{l}-1 \\
1\end{array}$ & $\begin{array}{l}-1 \\
1\end{array}$ & $\begin{array}{l}115 \\
112\end{array}$ & $\begin{array}{l}170 \\
171\end{array}$ & $\begin{array}{l}190 \\
197\end{array}$ \\
\hline $\mathrm{Mz}$ & $\begin{array}{l}1 \\
-1\end{array}$ & $\begin{array}{l}1 \\
-1\end{array}$ & $\begin{array}{l}1 \\
-1\end{array}$ & $\begin{array}{l}1 \\
-1\end{array}$ & $\begin{array}{l}1 \\
-1\end{array}$ & $\begin{array}{l}-8 \\
8\end{array}$ & $\begin{array}{l}-1128 \\
1128\end{array}$ & \\
\hline Mxy & $\begin{array}{l}- \\
-1\end{array}$ & $\begin{array}{l}- \\
-1\end{array}$ & $\begin{array}{l}- \\
1\end{array}$ & $\begin{array}{l}- \\
1\end{array}$ & $\begin{array}{l}- \\
-1\end{array}$ & 109 & 265 & 219 \\
\hline
\end{tabular}

${ }^{1}$ application of forces for this case is schematically shown in Fig. 2.

TABLE II: CRYO-LEG AND TIE ROD FORCES FOR DIFFERENT LOAD STEPS $(\mathrm{kN})$, (case Fxy in ANSYS).

\begin{tabular}{|c|c|c|c|c|c|c|c|c|}
\hline \multirow{2}{*}{$\begin{array}{l}\frac{0}{3} \\
\frac{0}{0} \\
\frac{8}{1} \\
\frac{1}{\frac{1}{0}} \\
\frac{0}{1}\end{array}$} & \multicolumn{2}{|c|}{ Cooldown } & \multicolumn{2}{|c|}{ HI } & \multicolumn{2}{|c|}{ HI \& TC (Fxy) } & \multicolumn{2}{|c|}{$\begin{array}{c}\text { HI \& TC (Fxy) - HI } \\
\text { (subtraction in ANSYS) }\end{array}$} \\
\hline & $\begin{array}{c}F_{\text {horizontal }} \\
\text { Tie-rod }\end{array}$ & $\begin{array}{c}F_{z} \text { (vertical) } \\
\text { Cryo-leg }\end{array}$ & $\begin{array}{l}F_{\text {horizontal }} \\
\text { Tie-rod }\end{array}$ & $\begin{array}{c}F_{z} \text { (vertical) } \\
\text { Cryo-leg }\end{array}$ & $\begin{array}{l}\mathbf{F}_{\text {horizontal }} \\
\text { Tie-rod }\end{array}$ & $\begin{array}{c}F_{z} \text { (vertical) } \\
\text { Cryo-leg }\end{array}$ & $\begin{array}{l}F_{\text {horizontal }} \\
\text { Tie-rod }\end{array}$ & $\begin{array}{c}F_{z} \text { (vertical) } \\
\text { Cryo-leg }\end{array}$ \\
\hline 10 & 2.52 & -500 & 28.8 & -64.5 & 28.6 & $-65,5$ & 0.366 & -1.00 \\
\hline 20 & 3.14 & -500 & 29.1 & -64.7 & 50.7 & \begin{tabular}{|l|}
-77.5 \\
\end{tabular} & 21.6 & -12.8 \\
\hline 30 & 3.01 & -499 & 29.1 & -64.2 & 42.9 & -49.3 & 13.7 & 14.9 \\
\hline 40 & 3.10 & -501 & 28.9 & -64.4 & 17.8 & -64.1 & 11.0 & 0.267 \\
\hline 50 & 3.21 & -501 & 32.1 & -64.9 & 38.6 & -73.5 & 6.59 & -8.61 \\
\hline 11 & 1.96 & -437 & 56.9 & -868 & 30.2 & -865 & 26.7 & 2.47 \\
\hline 21 & 2.06 & -437 & 56.7 & -868 & 17.5 & -852 & 39.2 & 16.1 \\
\hline 31 & 2.23 & -437 & 57.0 & -868 & 53.0 & -842 & 3.97 & 23.6 \\
\hline 41 & 1.95 & -437 & 57.4 & -869 & 80.8 & -888 & 23.4 & -19.3 \\
\hline 51 & 1.86 & -437 & 57.5 & -869 & 71.2 & -880 & 13.7 & -10.6 \\
\hline
\end{tabular}




\subsection{New design loads}

The 360. GM does not include components with masses without significant stiffness. Therefore a mass correction factor of 1.2 is modelled by corresponding increasing of the deadweight acceleration. As a result, additional compressive force is introduced on each cryo-leg in comparison with previous assessments published in [3]. Furthermore, pure FE results are multiplied by the factor 1.2 to cover FE simplifications [5]. This procedure and assuming the design friction factor of 0.1 in the bearings yields the forces as summarized in table 3 for the cyclic strength assessment of the cryolegs.

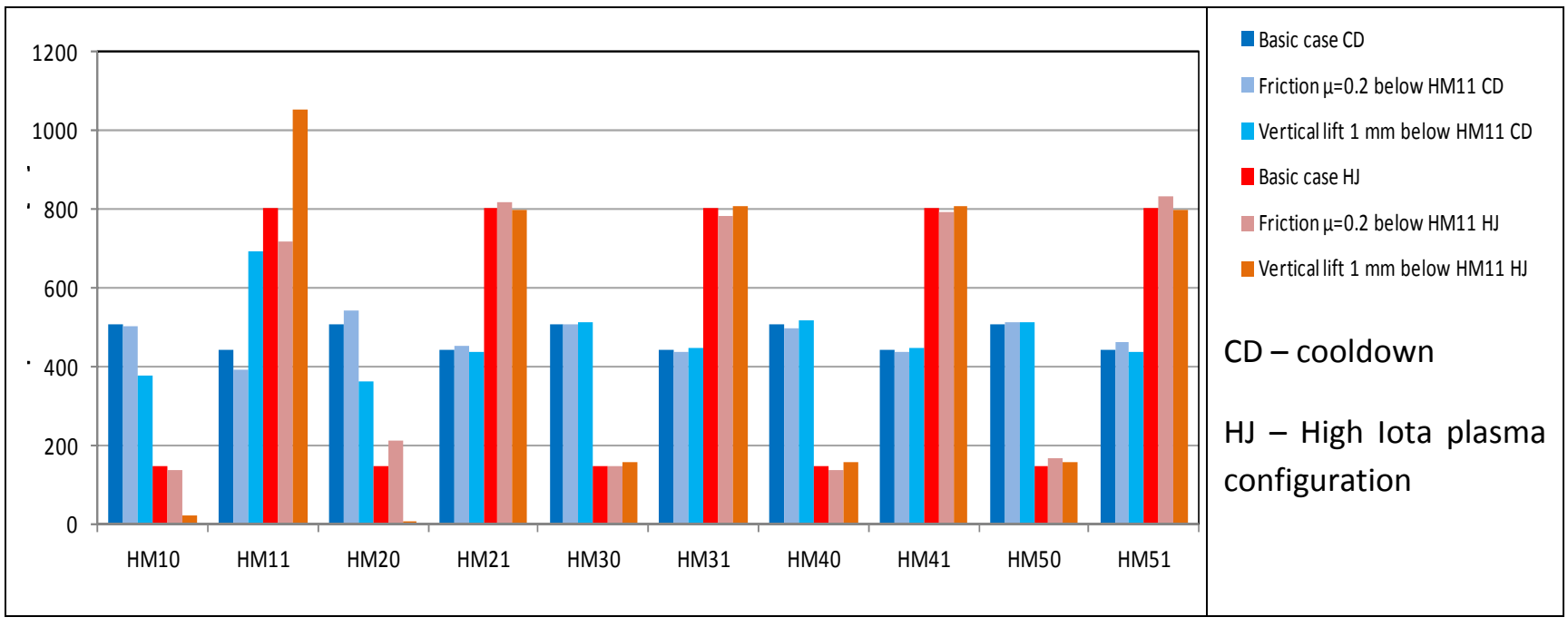

FIG.5. Asymmetry introduced in the magnet system monitored with cryo-leg vertical compressive forces (MN). Orange color corresponds to results of $1 \mathrm{~mm}$ cryo-leg raising in HM11.

ABAQUS FE model.

TABLE III: NEW LOAD CASES FOR FATIGUE LIFE ASSESSMENT (kN).

\begin{tabular}{|c|l|l|}
\hline Half module & Direction & Force \\
\hline \multirow{2}{*}{ HMx $1:$} & Compressive force & 1200 \\
\cline { 2 - 3 } & Horizontal force & 200 \\
\hline \multirow{2}{*}{ HMx0 } & Compressive force & $\sim 0 \div 10$ \\
\cline { 2 - 3 } & Horizontal force & 75 \\
\hline
\end{tabular}

\section{Cyclic strength of GRP tube}

\subsection{Test and assessment}

The GRP tube has been manufactured by IMA, Dresden, after optimization of R-Glass fibre winding directions in order to meet the high strength requirements [2]. In addition, a test with 1250 cycles of the original case 1 design forces ( $1 \mathrm{MNvert} / 156 \mathrm{kNhor}$ ) has been successfully passed, as well as 50 thermal cycles between RT and $77 \mathrm{~K}$. The manufacturer provided safety factors calculated by special 
software for each critical point of the GRP tube, including the drilled holes for the thermal anchor attachment [9]. A minimal safety factor of 1.2 was reported by IMA for the shrink-fitted end of the GRP tube which was squeezed between two steel rings (see figure 1). The stress level in that region is not influenced by cyclic loading of the tube, so the safety factor is sufficient.

A drilled hole in the middle of the tube is an obvious stress concentrator. The delamination between fibre and epoxy is possible in its vicinity, but the safety factor for the fibres is still above 4.5 [9]. Bending tensile stresses ( 20MPa) in the original case 2 design loads result in fibre delamination after a first loading due to the fact that a compressive vertical force of $10 \mathrm{kN}$ is not able to suppress bending generated by a horizontal force of $50 \mathrm{kN}$. However, no structural problems are expected due to the high safety factor for the fibre rupture $(>20)$. The new load case with almost zero compression of the cryo-leg requires additional consideration and detail analysis using the special IMA software or an equivalent one. This investigation is being initiated. In any case, compressive force in cryoleg can be increased, if necessary to mitigate the problem, by raising the warm cry-leg support as described in section 2.2. Further cyclic assessment of the GRP tube has been performed with the well-known idea that fatigue $S-N$ curve (alternating load/stress amplitude versus number of cycles to failure) can be mapped by a straight line in the log-log diagram:

$$
F(n) \approx R n^{C r}
$$

where $F$ is the load, $R$ the material strength limit for the static load, $n$ the number of cycles and $\mathrm{Cr}$ the characteristic coefficient of the GRP. The material strength limit $(R)$ was chosen to keep at least a factor of 5 against break of fibre in the GRP tube, and a factor of 2 against break of fibre in the drilled holes region. The estimation curve for the original case 1 design loads with a typical conservative value of $\mathrm{Cr} \sim-0.08$ is shown in figure 6.

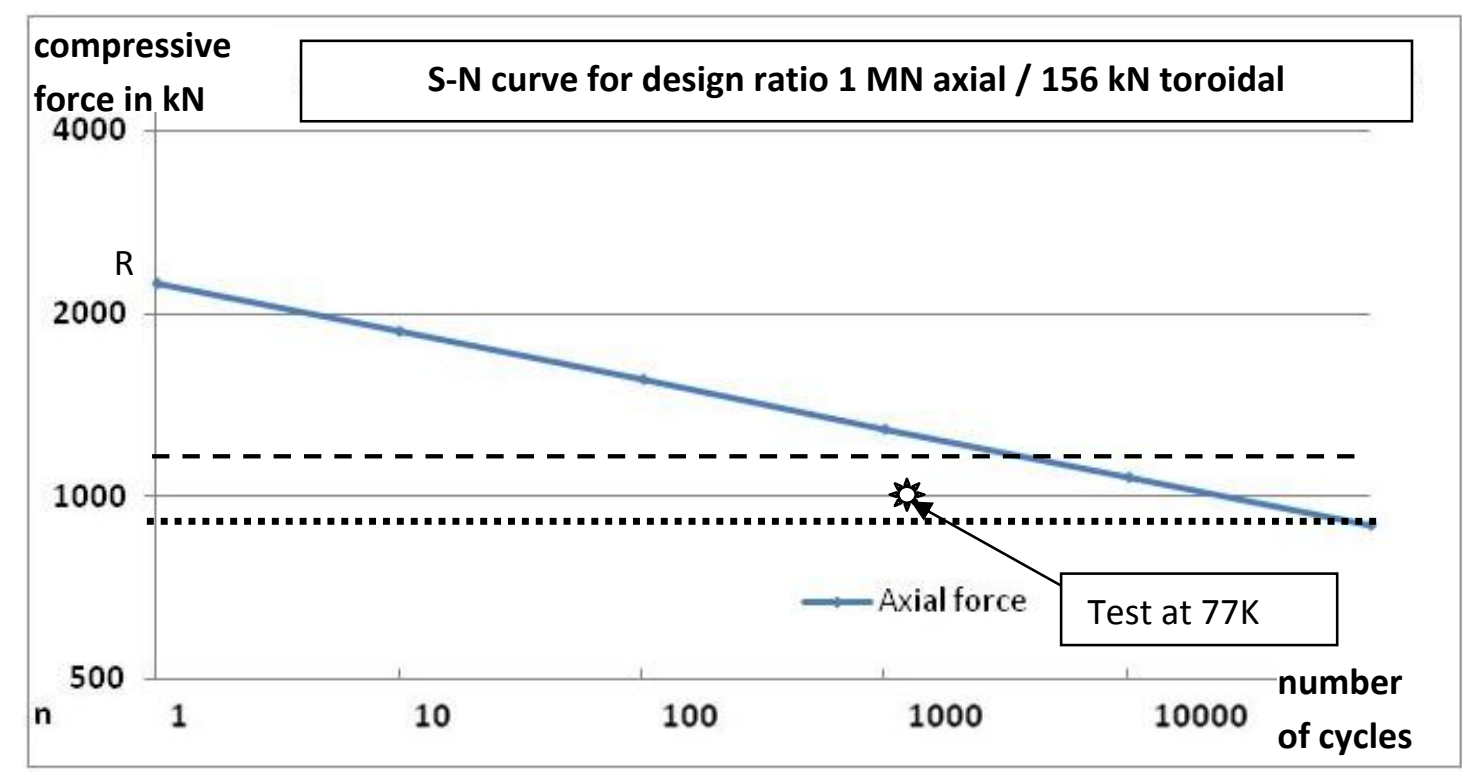

FIG. 6. Estimation fatigue S-N curve for case 1 design ratio (no safety factor on number of cycles).

\subsection{Discussion}

The fatigue curve shows clearly that the new load case cycles do not result in structural problems for the cryo-legs for the HMx1 loads according to table 3 (see dashed line on figure 6 ) up to $\approx 3000$ cycles. Present results of error field analysis [8] show that the maximal compensation capacities of 
the trim coils are not necessary. The probability is very low that error field compensation requires such extreme trim coil energizing that the EM forces generated are mainly in such unfavourable direction as given in table 1. Moreover, the friction factor 0.1 in the bearings is also an overestimation which generates such high loads. The expected value confirmed by the test is $<0.05$ [2]. The planned physics program considers that the number of $3 \mathrm{~T}$ cycles is not more than $10 \%$ of the total cycle number [10]. That basically means that only 6000 trim coil cycles are expected at the 3 $T$ field level. The estimation curve presented in figure 6 suggests that $\sim 600$ extreme trim coil cycles with a safety factor of 5 on the cycle number are not a problem for the GRP tube. One can safely assume that the operational cases with the mentioned simultaneous unfavourable conditions are far below this value.

The EM forces during 2.5 T operations are only $70-80 \%$ of the considered ones. That allows to accept theoretically almost 100000 cycles, or $\sim 20000$ extreme trim coil cycle with a safety factor of 5 at the $2.5 \mathrm{~T}$ field level (see dotted line on figure 6).

In any case, a cryo-leg monitoring system is installed as briefly described in the next chapter. Moreover, lifting of the cryo-leg is considered as a failure and will be avoided by correction of the vertical position of the corresponding cryo-legs.

Nevertheless, a refined assessment of the GRP tube under mainly horizontal cyclic loads (HMxO in table 3 ) is being launched.

With the original quasi-static design loads the stress concentrations at the through holes in the GRP tube for anchoring the thermal shield of the outer cryostat vessel were no problem. The bolts through these holes were also used to fix and thermally connect a circular thermal shield plate at the inside of the cryo-leg tubes. With the additional new loads the stresses come closer to the limits as described.

Shrink-fit technology for the attachment of the thermal anchor from inside and outside would be a possible solution to eliminate the problem. Reinforcement the shield contact area, or gluing/clamping solutions could be other options.

The CSS in form of a five-periodic ring deforms wave like in all directions under EM loads. The wave length of the deformations is one toroidal module length. The cryo-legs are installed in regions with considerable derivation of this sinus-like wave. Therefore, the deformation of the CSS imposes cryoleg tilting in opposite directions.

Positioning of only five cryo-legs in areas of sinus wave extremes would have largely avoided tilting with the consequences of additional MS shifts [3] and sliding in the bearing as well as significant shear forces and bending moments in the cryo-legs.

\section{Cryo-leg behaviour monitoring}

Since the cryo-legs are critical components for the W7-X MS operation, the bottom part of all ten supports and tie-rods is equipped with strain gages and displacement sensors [11]. The continuous monitoring will be provided starting from cool-down through commissioning of the coil groups and during all stages of operation. These sensor signals, together with those from other mechanical instrumentation, will permanently be recorded and stored in the data archive and can easily be 
retrieved for further analyses. Sliding during first cool-down of the MS will be equipped with independent instrumentation to benchmark signals from the permanently installed displacement sensors.

In case that sliding of a cryo-leg does not occur as expected for some currently unknown reason, a procedure is prepared to push it from outside if the movement lags by more than $2 \mathrm{~mm}$ w.r.t. the predicted value. In case a cryo-leg becomes unloaded close to lift-off, the warm support on the MB will be raised correspondingly.

\section{Conclusions}

The integrity of the complex W7-X MS structure has been confirmed by two independent FE models. The advanced analyses considering unsymmetrical cryo-leg loads due to unavoidable assembly inaccuracies and EM forces generated by trim coils indicate that maximally possible forces are well above the original design loads. This means that the number of trim coil cycles needs to be considered, counted and assessed in the course of W7-X operation based on cryo-leg behavior monitoring and FE analyses. In any case, no serious trim coil operational limitations are expected.

\section{References}

[1] Tamura H. et al 2007 Design and optimization of support post for cryogenic component in FFHR ITC/ISHW2007: Proc. 17th Int. Toki Conf. and 16th Int. Stellarator/Heliotron Workshop (Toki, Japan, 15-19 October 2007)

www.nifs.ac.jp/itc/itc17/file/PDF proceedings/ poster1/P1-094.pdf

[2] Jenzsch H. et al 2008 Final design and manufacturing of the cryolegs of the W7-X-superconducting coil support system Fusion Eng. Des. 83 1600-4

[3] Egorov K., Bykov V., Schauer F. and Van Eeten P. 2009 Structural analysis of Wendelstein 7-X magnet weight supports Fusion Eng. Des. 84 722-8 www.sciencedirect.com/science/article/pii/ S0920379609000556

[4] Freundt S. et al 2013 FE analyses and tests in support of Wendelstein 7-X trim coil development Fusion Eng. Des. 88 1589-92

[5] Bykov V., Fellinger J., Schauer F., K“oppen M., Egorov K., Van Eeten P., Dudek A. and Andreeva T. 2014 Specific features of Wendelstein 7-X structural analyses IEEE Trans. Plasma Sci. 42 690-7

[6] Bykov V. et al 2014 Advanced structural analysis of Wendelstein 7-X magnet system weight supports FEC2014: Proc. 25th IAEA Fusion Energy Conf. (St Petersburg, Russia, October 2014) https://conferences.iaea.org/

indico/contributionDisplay.py?contribId=113\& sessionId=34\&confId $=46$

[7] Fellinger J. et al 2014 Asymmetry of W7-X magnet system introduced by torus assembly Fusion Eng. Des. 89 2189-93

[8] Andreeva T., Br"auer T., Bykov V., Egorov K., Endler M., Fellinger J., Kißlinger J., K“oppen M. and Schauer F. 2015 Tracking of the magnet system geometry during Wendelstein 7-X construction to achieve the designed magnetic field Nucl. Fusion at press [9] 2008 Entwicklungsbericht Cryoleg Report B297/07-2, IMA Materialforshung und Anwendungstechnick GmbH

[10] Fellinger J., Bykov V. and Schauer F. 2013 Assessment of cracks in lateral supports of the magnet system of Wendelstein 7-X Fusion Eng. Des. 88 1465-8

[11] Fellinger J., Egorov K., Bykov V. and Schauer F. 2015 Assessment of instrumentation of magnet system of Wendelstein 7-X Fusion Eng. Des. at press 